\title{
October 2015 Critical Care Case of the Month: A Moldy But Gooey
}

\author{
Jennifer M. Hall, DO \\ Banner University Medical Center Phoenix \\ Phoenix, AZ
}

\section{History of Present IIIness}

A 45-year-old man with a history of a kidney transplant in 2011 was admitted with subjective fevers, nausea, abdominal pain, chest pain and recurrent renal failure. Cardiac workup was negative for ischemia and intermittent hemodialysis was initiated. CT of chest and abdomen was significant for a new cavitary pulmonary lesion. Leading up to this admission, he had been on immunosuppressive agents including tacrolimus, mycophenolate and prednisone, and the day of presentation had been doing quite well, actually was bear hunting in the mountains near Flagstaff, Arizona.

\section{Past Medical History}

- Donor kidney transplant in 1999 , which failed in 2011, prompting a second kidney transplant

- Failed pancreas transplant

- Coronary artery disease, with percutaneous cardiac intervention in 2001

- Diabetes mellitus type I

- Chronic anemia

- History of total parathyroidectomy

- History of C5-C7 cervical fixation

\section{Physical Examination}

- Vital signs stable

- Appeared to be pale, no apparent distress

- Cardiac exam unremarkable

- Chest exam with fine crackles in left base / otherwise clear

- Abdomen slightly tender in left lower quadrant, but without guarding, rebound or peritoneal signs; small dime-sized area of ecchymosis, where lovenox injections had been administered

- No peripheral edema or clubbing

\section{Laboratory Evaluation}

- WBC 17,900 cells/mcL with $96 \%$ segmented neutrophils, hemoglobin 8.9 g/dL(after transfused 2 units prior to transfer), PLT 232,000 cells/mcL,

- $\quad$ INR 1.3 
- Blood urea nitrogen (BUN) $74 \mathrm{mg} / \mathrm{dL}$, serum creatinine $2.32 \mathrm{mg} / \mathrm{dL}$, electrolytes within normal limits, albumin $3.2 \mathrm{~g} / \mathrm{dL}$, aspartate aminotransferase (AST) $24 \mathrm{IU} / \mathrm{L}$, alanine transaminase (ALT) $81 \mathrm{IU} / \mathrm{L}$.

- NT-proBNP $6841 \mathrm{pg} / \mathrm{ml}($ normal $<300 \mathrm{pg} / \mathrm{ml})$

- Hemoglobin A1C 7.2\%

- Lactate $0.7 \mathrm{mmol} / \mathrm{L}$

\section{Imaging}

A thoracic CT scan was performed (Figure 1).

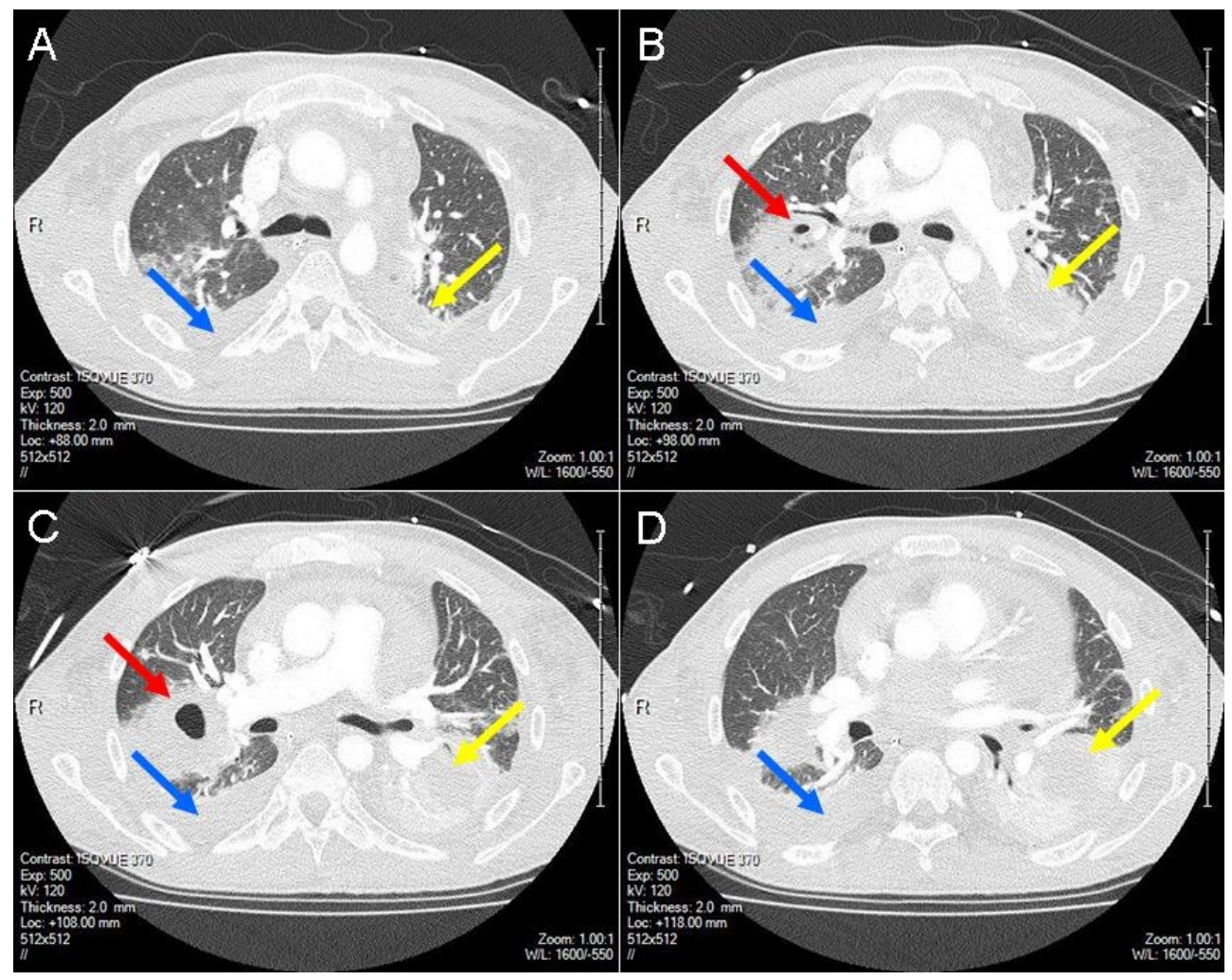

Figure 1. Representative static views from the CT scan in lung windows. Note the cavitary lesion in the right lung (red arrow), the right pleural effusion (blue arrow) and the left lower lobe consolidation (yellow arrow) with a pleural effusion.

Which diagnosis is least likely in this patient's differential diagnosis for the cavitary pulmonary lesion?

1. Aspergillosis

2. Coccidioidomycosis

3. Invasive mucormycosis

4. Metastatic malignancy

5. Nocardiosis

6. Pulmonary Infarct 


\section{Correct! \\ 4. Metastatic malignancy}

Metastatic malignancy is not likely given an isolated cavitary lesion. Certainly one may find cavitary lesions associated with certain primary pulmonary malignancies, such as squamous cell carcinoma, primary lymphoma and lymphomatous granulomatosis, but with metastatic secondary malignancies, these characteristically present as hematogenous spread with multiple solid nodules. The other choices are within the differential, especially infections, and given his immunosuppressed state, one must consider fungal, atypical, mycobacterial and other opportunistic infections. There are other considerations including infarcts, septic embolic phenomena, autoimmune diseases, granulomatous processes, parasitic infections and some less common entities. But, in our patient, we were primarily focused on infections given his immunocompromised status.

The patient underwent bronchoscopy with bronchoalveolar lavage as well as endobronchial ultrasound and biopsy of an enlarged mediastinal lymph node. A chest tube placed for the left-sided pleural effusion. All of the serologies and cultures were negative, and pleural fluid was a neutrophilic-predominant exudative effusion, but with negative work up otherwise. Empirically he was started on fluconazole to cover for coccidioidomycosis, and then later in his course, voriconazole was added for the possibility of aspergillosis.

Clinically he had a gradual decline over the next couple of weeks including rising WBC, persistent fevers, worsening abdominal pain with distention and shortness of breath, prompting his transfer to the ICU. A CT of the abdomen and pelvis was obtained (Figure 2).

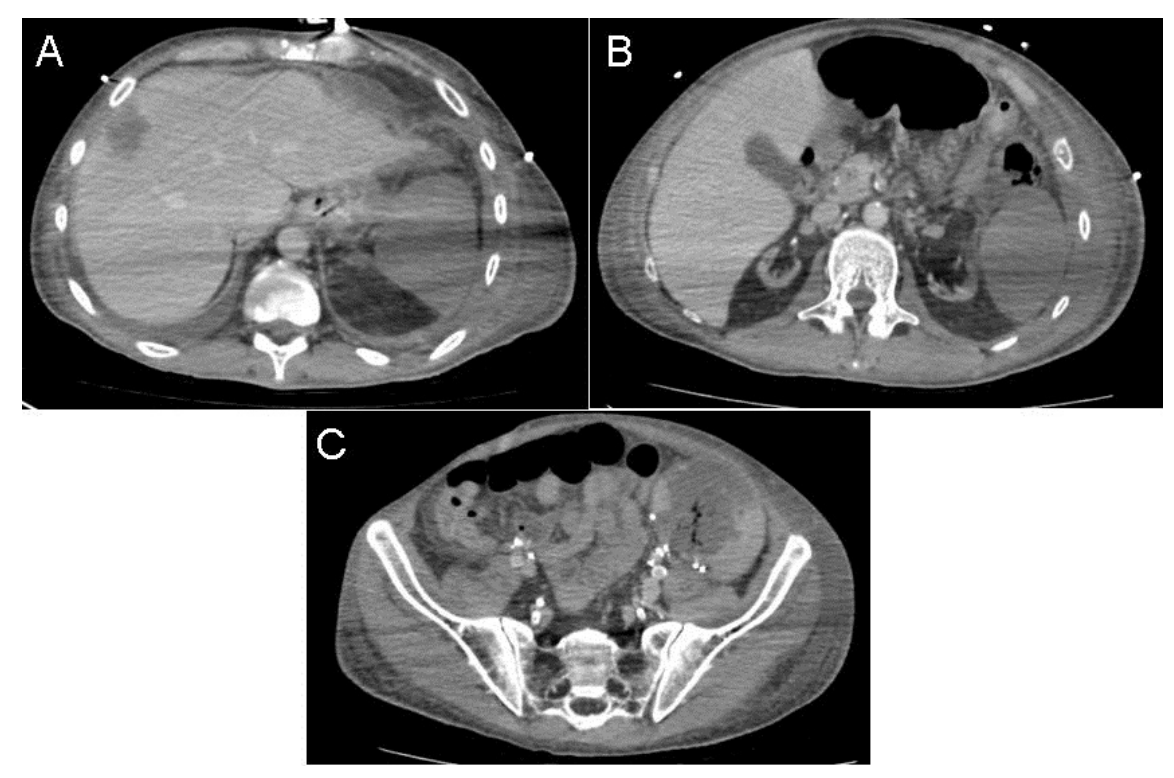

Figure 2. Selected views from abdominal CT scan consistent with multiple areas of infraction. 
There were no abscesses on the exploratory laparoscopy and also no evidence of perforation. The donor kidney was explanted, without evidence of infectious process, but revealing necrosis.

What would be the next best step in evaluation for patient's organ infarcts?

1. Antiphospholipid Syndrome (APLS) workup

2. Echocardiogram (TEE)

3. Liver biopsy

4. Splenectomy

5. $1 \& 2$

6. $3 \& 4$ 


\section{Correct!}

\section{5. $1 \& 2$}

Both TEE and workup for APLS should be considered. In the setting of multiple organ infarcts, one should think about septic emboli, cardiac embolic phenomena, mycotic aneurysms, hypercoagulable states, and vasculitic conditions. His blood cultures were repeatedly negative over the $>2$ week course, as well as all other negative cultures to date. Therefore, there was consideration for HACEK (Haemophilus, Aggregatibacter, Cardiobacterium, Eikenella corrodens, Kingella) organisms in endocarditis, fastidious bacteria such as Nocardia or Actinomyces, other infectious mycotic aneurysm, and fungal infections especially ones with poor sensitivity to growth on blood cultures. Additionally, a non-infectious embolic source also can present as in this case and should be entertained.

Performing a liver biopsy or splenectomy would be risky given the potential for septic spread and bleeding, and, if infarcts are present, there may be low yield. Furthermore, the donor kidney was explanted and thus performing another biopsy or obtaining pathology of another organ would probably be unnecessary and with added risk.

The patient did undergo TEE which was negative. APLS workup also was negative. We did perform a CTA to evaluated for mycotic aneurysm as well for progression of the cavitary lung lesion - these tests were negative. A few days later, after a period of defervescence, the patient returned again to the ICU with progressively worsening abdominal pain, a CT revealing increasing amount and size of organ infarcts as well as evidence of a lateral wall of myocardium appearing to be infarcted, and with signs and symptoms of an acute STEMI. He was urgently taken to the cardiac catherization lab revealing an occluded left circumflex of which he underwent PCTA, and then to follow, he was taken to the OR where another exploratory laparotomy was performed. The spleen was infarcted and there was an ischemic-appearing gastric cardia and small bowel and necrotic tissue in the retroperitoneum. No abscess, stool, purulence or enteric contents was noted in the peritoneal cavity, and the necrotic tissue was too friable and there would have been an elevated risk of bleeding for any surgical intervention.

CT was also concerning for a peri-splenic abscess, an area of newly loculated left pleural effusion and now with a skin lesion on his right abdomen with central necrosis and a rim of dusky mottled appearance. Drains were placed both in the peri-splenic abscess and in the pleural effusion, with growth of GNR, and following the drains, a skin biopsy was performed.

Patient decompensated later that evening, progressing to a state of shock and died later that night. The fluid grew Bactericides fragilis and Rhizopus species. The skin biopsy was positive for fungal elements resembling mucoracae. And the final autopsy report confirmed the diagnosis of disseminated mucormycosis with associated multi-organ infarcts with noted fungus with each organ involved. 
What is the best first-line anti-fungal treatment for mucormycosis?

1. Fluconazole

2. Liposomal amphotericin B

3. Micafungin

4. Posaconazole

5. Voriconazole 


\section{Correct! \\ 2. Liposomal amphotericin B}

Liposomal amphotericin B is the first line drug of choice if one is diagnosed with or even if there is a strong suspicion for mucormycosis. The liposomal formulation has been much better tolerated and less risk to the kidneys. Posaconazole may also be used, but this is not first line. Typically, this anti fungal agent is either a substitute for poor tolerance to amphotericin, or may be used as adjunctive therapy for mucormycosis. The other anti fungal agents listed have NO activity against mucoracae, except for micafungin, which has minimal activity against this fungus; but you would not employ this agent as it is only weakly effective and does not cure this fungal disease. Given its $100 \%$ fatality rate if untreated, one must use the appropriate therapy as mentioned.

Surgical resection, if feasible, is also considered first line, in particular with rhinocerebral mucormycosis, but there is limited evidence of benefit with invasive / disseminated disease.

Our patient may have benefited from early amphotericin, however, at the time we saw him in the ICU, this process had already disseminated, and thus likely was futile from the start. We failed to see the connection in his disease process, underlying immunocompromised state and the possibility for invasive / disseminated mucormycosis.

What would have been the best test to obtain a positive diagnosis for mucormycosis and with best sensitivity?

1. 1,3-B-D-glucan assay

2. Fluid cultures run on chocolate agar

3. Fungal blood cultures

4. Galactomannan antigen from BAL

5. Tissue biopsy from affected organ or skin 


\section{Correct! \\ 5. Tissue biopsy from affected organ or skin}

The gold standard for diagnosing mucormycosis is a tissue biopsy and histopathology revealing the characteristic ribbon-type morphology with 45 degree branching of fungal elements. 1,3-B'D glucan and galactomannan are negative in the case of mucormycosis, but rather are tests that one would consider obtaining for evaluation for aspergillosis. Both of these tests were negative or this patient, thus indicating a much less likelihood for aspergillosis and giving a clue for another disease process, in particular another invasive fungal process. Blood cultures for mucormycosis are invariable negative with poor sensitivities, likely due to the fact that the fungal walls can easily be disrupted, and often one would need to prepare the specimen with fine cuts rather than a process that breaks apart the integrity of the fungal wall.

\section{Learning Points}

- Early diagnosis and treatment of mucormycosis is one cornerstone to successfully managing this aggressive disease.

- Immunocompromised hosts are at the highest risk for mucormycosis, especially with solid organ transplant recipients and hematological malignancies with hematopoietic stem cell transplantation.

- One should consider mucormycosis in any immunocompromised host, especially if fungal infection is a concern.

- When mucormycosis has disseminated, this represents its angioinvasive form which typically results in hemorrhage, necrosis and infarctions. At this stage, it is invariably fatal.

- The diagnostic test of choice is to obtain a tissue biopsy.

- Treatment of choice is liposomal amphotericin B

\section{Reference}

1. Hamdi T, Karthikeyan $\mathrm{V}$, Alangaden GJ. Mucormycosis in a renal transplant recipient: case report and comprehensive review of literature. Int J Nephrol. 2014;2014:950643. [CrossRef] [PubMed] 